\title{
Alterstice
}

Revue internationale de la recherche interculturelle

International Journal of Intercultural Research

Revista International de la Investigacion Intercultural

\section{Théories et pratiques de l’interprétation de service public, de Sophie Pointurier}

\section{François René de Cotret}

Volume 6, numéro 2, 2016

URI : https://id.erudit.org/iderudit/1040633ar

DOI : https://doi.org/10.7202/1040633ar

Aller au sommaire du numéro

Éditeur(s)

Alterstice

ISSN

1923-919X (numérique)

Découvrir la revue

Citer ce compte rendu

René de Cotret, F. (2016). Compte rendu de [Théories et pratiques de

l'interprétation de service public, de Sophie Pointurier]. Alterstice, 6(2), 71-75.

https://doi.org/10.7202/1040633ar d'utilisation que vous pouvez consulter en ligne.

https://apropos.erudit.org/fr/usagers/politique-dutilisation/ 


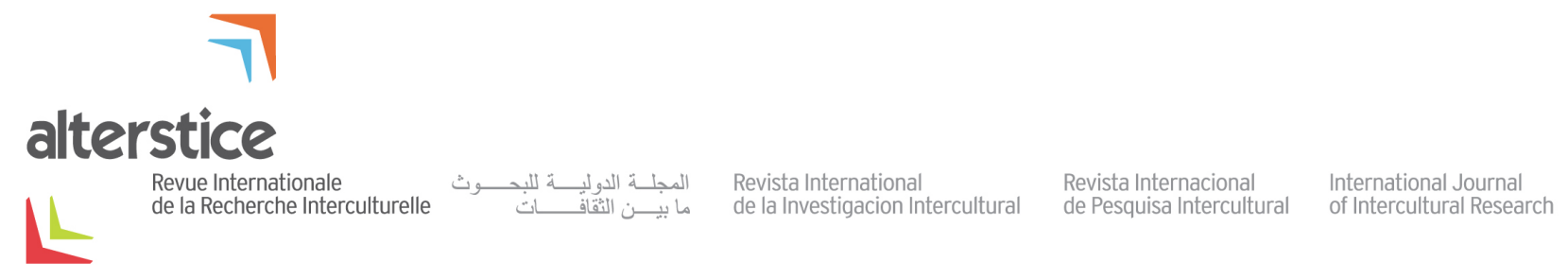

NOTE DE LECTURE

\title{
Théories et pratiques de l'interprétation de service public, de Sophie Pointurier
}

François René de Cotret $^{1}$

\author{
Rattachement de l'auteur \\ ${ }^{1}$ Université Laval, Québec, Canada
}

\section{Correspondance}

francois.rene-de-cotret.1@ulaval

\section{Références de l'ouvrage}

Pointurier, S. (2016). Théories et pratiques de l'interprétation de service public. Presse Sorbonne Nouvelle. Paris, France.

\section{Pour citer cet article}

René de Cotret, F. (2016). Théories et pratiques de l'interprétation de service public [Note de lecture]. Alterstice, 6(2), 71-75.

\section{Introduction et présentation}

Un seul après-midi m'aura été nécessaire pour dévorer le premier livre de Sophie Pointurier, un essai d'un peu plus de 100 pages. De l'Antiquité à la mondialisation en passant par le traité de Versailles - qui coiffe la transition du français vers l'anglais comme langue internationale, ai-je appris - l'auteure donne de précieuses clés au lecteur en quête de (nouvelles) connaissances à propos d'un métier millénaire des plus humbles, l'interprétariat. J'utilise ici le terme " interprétariat » pour référer à la fonction ou au métier de l'interprète, ce que Sophie Pointurier nomme « interprétation». Dans cette note de lecture, je restreins l'emploi du terme "interprétation » à l'action de traduire oralement.

La double formation d'interprète et de chercheure à la Sorbonne Nouvelle, où elle dirige le Master d'Interprétation de la langue des signes française de l'École supérieure d'interprètes et de traducteurs, fait du docteure Pointurier la personne tout indiquée pour instruire aussi bien le chercheur que l'interprète de métier. Le livre offre d'ailleurs deux sections : on aborde des notions théoriques tirées de sources scientifiques considérables, pour ensuite passer à des applications pratiques dans trois contextes publics : judiciaire, médical et pédagogique. Bien qu'on y retrouve un nombre de réflexions originales à propos de la langue des signes, je vais restreindre, par souci de concision, ma synthèse à l'interprétariat en général. 
La première section débute avec une définition ainsi qu'une typologie de l'interprétation, des clarifications permettant de départager un vocabulaire parfois escamoté dans la littérature scientifique. L'interprétation, explique l'auteure en citant Kade de l'école de Leipzig, appartient au domaine de la traduction et requiert deux conditions : (a) un texte source présenté une seule fois ainsi que (b) la contrainte du temps, avec une impossibilité de rectifier le tir. Tenir compte de la modalité d'interprétation, on le remarquera tout au long du livre, importe également : en simultané, en chuchoté, à vue, à distance, en visioconférence, etc.

En s'appuyant sur Pöchhacker, $M^{\text {me }}$ Pointurier pose que l'interprétariat d'aujourd'hui implique une migration de plus en plus marquée de "l'intersocial (entre plusieurs sociétés) vers l'intrasocial (au sein même d'une seule société) » (p. 30). L'interprétariat de conférence, la forme la plus connue et historiquement synonyme de grandes rencontres internationales, perdrait du terrain au profit d'une autre forme, qu'elle nomme l'interprétariat en service public. Cette appellation forme un carrefour où se rencontrent interprétariat ou médiation sociale, interprétariat communautaire, interprétariat de liaison, Public Service Interpreting, Multicultural Communication, Liaison Interpreting et Community Interpreting :

[Cette forme d'interprétariat] est envisagée ici dans son sens le plus noble, c'est-à-dire l'accessibilité des structures mises à disposition de tous les citoyens. C'est un domaine de recherche récent, dont l'intérêt va croissant au fil des évolutions législatives qui instaurent des droits relatifs à l'accessibilité des personnes étrangères et/ou en situation de handicap (surdité). (p. 31)

\section{Schématiser l'interprétation}

Avant la Seconde Guerre mondiale, les théoriciens avaient tendance à considérer le texte à traduire comme seul paramètre de la traduction. Avec l'émergence de la traductologie, la manière de considérer cette pratique s'est nuancée, et d'autres paramètres ont été envisagés: traducteur, destinataires de la traduction, contexte dans laquelle elle s'inscrit, etc.

Les années 1960 ont vu apparaître la Théorie interprétative de la traduction, qui a comme premier principe la recherche de sens, par opposition à la recherche pure et simple d'équivalences linguistiques entre les langues. Voici l'exemple donné dans le livre pour saisir l'idée :

[L]orsqu'une personne entre dans une pièce en s'adressant à une autre en lui disant qu'il fait froid ici, cela veut peut-être signifier qu'elle aimerait bien que l'on ferme la fenêtre. Pourtant, ni le mot "fermer " ni celui de "fenêtre " n'ont été prononcés dans la phrase. II fait froid ici veut dire dans ce contexte Je voudrais que tu fermes la fenêtre ou Pouvons-nous monter le chauffage. (p. 43)

On comprend dans cet exemple que la signification du message ne se retrouve pas uniquement dans les mots, mais également dans les connaissances extralinguistiques de l'interprète et de sa compréhension du contexte dans lequel il intervient. Ainsi, il n'est pas étonnant de voir un interprète accomplir son travail plus aisément lorsqu'il a l'opportunité de se préparer avant un entretien ou de suivre des formations pour enrichir ses connaissances à propos de son milieu de pratique.

La théorie du skopos, popularisée dans les années 1980 et 1990, minimise également la notion d'équivalence linguistique. Skopos, un mot grec signifiant "finalité ", réfère à un principe de la théorie éponyme, soit de déterminer l'action de la traduction, c'est-à-dire sa finalité, sa fonction. Selon cette théorie, l'évaluation de la qualité de la traduction passe notamment par la compréhension des besoins du récepteur de la traduction et de la réussite de cet objectif. Par exemple, interpréter pour un patient, pour un médecin ou pour les deux, pour l'établissement de santé ou pour le bien-être du patient aurait une incidence sur le message transmis.

La docteure Pointurier présente également les modèles pédagogiques de son directeur de thèse, Daniel Gile. Ces modèles sont inspirés du concept de ressources attentionnelles, cher à la psychologie cognitive : il est entre autres question d' " énergie mentale ", dont les quantités, limitées, seraient à la merci de certains paramètres. Parmi ceuxci se trouvent la connaissance du sujet par l'interprète, son état de stress ou de fatigue et les conditions de travail 
(bonne acoustique/visibilité, limite de temps, etc.). C'est en considérant ces paramètres que Gile introduit la notion d'« Efforts », sous-ensembles d'opérations cognitives qui participent à l'interprétation :

- Effort de Réception;

- Effort de Mémoire (à court terme pour retenir puis récupérer l'information);

- Effort de Production (du discours en langue cible);

- Effort de Coordination (de l'affectation des ressources attentionnelles entre les différents efforts afin d'assurer l'interprétation).

Une telle schématisation permet de comprendre la portée de l'équilibre entre les différents Efforts ainsi que la conséquence d'une trop grande demande pour un Effort donné, soit la détérioration de la qualité de l'interprétation. Pour expliquer à quel point la mémoire de travail des interprètes est sollicitée, Gile a développé "l'hypothèse de la corde raide », voulant que le total de l'attention requise pour l'interprétation soit généralement proche de la capacité attentionnelle maximale de l'interprète. Le facteur temps est également déterminant dans ces modèles. Par exemple, lorsque la vitesse de production est moins rapide que celle du discours, l'Effort de Mémoire devient rapidement trop important. L'auteure précise :

C'est souvent dans les segments de discours qui suivent un segment difficile, et non pendant celui-ci, que la capacité de traitement est saturée. (p. 54)

À la différence de l'interprétation de conférence, effectuée à sens unique, l'interprétation de service public est dialogique, ce qui a comme conséquence, suppose l'auteure, d'ajouter passablement de stress sur la mémoire de travail de l'interprète. À cela s'ajoute notamment le contexte affectif de l'entretien, parfois effectué en situation d'urgence ou de détresse, et le fait que l'interprète doit gérer aussi le discours qui lui est directement adressé lors de l'entretien. La liste des variables ne s'arrête pourtant pas ici.

\section{D'autres variables dans l'équation}

Comprendre l'interprétariat, c'est aussi tenir compte des facteurs d'ordre sociologique qui se trouvent dans l'équation. Lors de l'entretien, l'interprète est appelé à remplir des rôles extralinguistiques tels qu'éclaircir certains points, soulever les ambiguïtés du discours en mettant en mots les non-dits, déterminer les tours de parole et respecter les normes sociales attendues des locuteurs.

En se référant à Saleskovitch et Wadensjö, l'auteure introduit la notion de coopération. Elle explique que l'interprète produit deux types de discours : un premier relevant de la transmission translinguistique du contenu, l'autre relevant de la coordination, nécessaire pour maintenir le flux de conversation des interlocuteurs. Sa capacité à coordonner l'interaction serait ainsi déterminante. Par exemple, l'interprète est censé gagner la confiance des locuteurs pour être en mesure de maintenir une relation entre ceux-ci sans accaparer l'entretien, ce qui demande de l'assurance personnelle ainsi que de bonnes capacités communicatives et sociales.

La notion de groupe social, rattachée aux normes de communication, doit aussi être prise en compte par l'interprète. Par exemple, la position sociale du migrant, en situation de détresse ou d'inconfort physique, dans une situation de vie précaire, n'est pas celle du médecin, jouissant d'une certaine notoriété et se positionnant en tant que détenteur d'un savoir. L'interprète doit ainsi tenir compte de normes sociales propres à certains groupes et à certaines situations afin de s'assurer de maintenir les locuteurs dans une zone de confort communicationnelle, loin d'une expérience déroutante.

Contrairement à l'interprète de conférence, qui est au cœur des événements historiques, l'interprète de service public est au cœur d'événements vécus par des citoyens. $\mathrm{M}^{\text {me }}$ Pointurier emprunte le terme " vicarious trauma » de C. Figley pour mettre en lumière les possibles retombés de l'entretien sur l'interprète. L'empathie envers le client, le concept d'identification à l'autre et les mécanismes impliquant les neurones miroirs sont autant de facteurs psychologiques pouvant occasionner une dérive émotionnelle. 
Le souci d'impartialité - que plusieurs confondent encore avec la neutralité -, ne pourrait protéger l'interprète de ladite dérive, insiste-t-elle, car l'impartialité ne signifie pas l'absence de réaction émotionnelle. De plus en plus populaire en Europe, la supervision de l'interprète, à l'image de la supervision d'autres professionnels comme le psychologue, semble prometteuse :

Il s'agit de proposer [...] un moment totalement dédié à l'écoute, à la parole et à l'analyse de [ses] pratiques et de [ses] éventuelles conséquences sur [son] état psychologique général ». (p. 75)

La supervision servirait également à identifier les moments de l'entretien lors desquels l'interprète peut être porté à prendre parti. Même si l'interprète revendique l'impartialité, la recherche démontrerait qu'il n'est pas à l'abri de prises de position, par exemple en encourageant une parole plus que l'autre ou en ne traduisant pas nécessairement un segment.

L'auteure complète la notion d'impartialité avec celle de la double alliance, signifiant que l'interprète doit être avec l'un aussi bien qu'avec l'autre. Cette manière de concevoir l'entretien permet à l'interprète de s'autoriser à intégrer certaines de ses prises de position à tour de rôle, au lieu de se réfréner, " pour servir au mieux le vouloir-dire et les intentions de chaque interlocuteur » (p. 87). Cela rejoindrait le paradoxe de la neutralité : l'interprète minimise son impact dans le discours du moment qu'il prend conscience de son rôle dans l'interaction et l'assume.

\section{Éthique et déontologie}

En s'appuyant sur plusieurs codes d'interprétariat, $M^{\text {me }}$ Pointurier développe six principes fondamentaux permettant de réguler la relation entre l'interprète et ses clients et d'assurer sa qualité : le secret professionnel, la fidélité, l'impartialité, la discrétion, la solidarité et la probité. Elle note cependant que les présentations actuelles des codes, outre le fait qu'elles sont généralement tirées d'une réflexion relevant de l'interprétariat de conférence, sont généralement infantilisantes pour l'interprète, à qui on dicterait de ne pas faire telle chose ou telle autre. À ses yeux et à ceux des auteurs qu'elle cite, tels qu'Angelelli et Shlesinger, une évolution déontologique et éthique est nécessaire, afin « d'offrir un cadre respectueux du travail effectif de l'interprète et de minimiser son impact sur l'interaction » (p. 90).

Certains des changements qu'elle juge nécessaires, en particulier la révision du principe d'impartialité, traversent son livre. Avant de passer à la section des applications pratiques, elle traite par exemple du principe de fidélité et des questions qui l'entourent. Dans le contexte de service public, à qui est-ce que l'interprète devrait fidélité ? Au message ? À un ou l'autre des locuteurs ou à tous les locuteurs ? À sa propre éthique?

\section{Applications pratiques}

La seconde section regorge d'outils destinés à l'interprète de service public. Par exemple, par rapport à l'étape de la préparation à un entretien, on suggère de suivre la règle des cinq questions en journalisme : "Qui ? Quand ? Quoi ? Où ? Comment ? ". Les réponses devraient permettre un enlignement aussi bien du côté terminologique que conceptuel et psychologique. Autre exemple : par rapport à la présentation en début d'entretien, on fait allusion à la nécessité d'instaurer un rapport de confiance, dont il a été question auparavant, en poursuivant avec quelques règles de base.

Également, l'auteure décortique trois contextes particuliers d'interprétation (judiciaire, médical et pédagogique) afin de permettre à l'interprète de s'adapter autant que possible aux différentes réalités propres à chacun d'eux. Tirée de Leanza et Boivin, sa réflexion quant au pouvoir de la parole dans le domaine médical est particulièrement éclairante. En tant qu'expert, le médecin détient un savoir et donc un pouvoir, qui est appelé à transiter par l'interprète. Cette idée de partage du pouvoir - ou le rejet de celle-ci par le médecin - pousse à réfléchir sur ce qui est en jeu ici, aussi bien sur le terrain que cristallisé dans les codes éthiques et déontologiques. Est-ce que cette contrainte du transfert de pouvoir peut expliquer, ne serait-ce que partiellement, la force avec laquelle l'institution médicale insiste sur le principe de neutralité de l'interprète ? Expliquerait-elle les difficultés rencontrées par celui-ci dès qu'il essaie de s'affirmer en tant qu'expert de l'interprétation (en faisant autre chose qu'une interprétation mot à mot) ? 


\section{Notre République}

Accueillir l'Autre "dans la République", pour reprendre les mots de l'auteure, est certainement un des grands défis de notre époque, marquée par des mouvements humains sans égal. Suivre l'esprit du temps, elle nous le démontre habilement, passera entre autres par l'interprétariat de service public. Chemin faisant, elle livre un message porteur de promesses pour l'interprète, et d'opportunités pour le chercheur du domaine, mais également chargé d'un devoir politique aussi bien pour l'un que pour l'autre. Une discussion limpide entre ces deux acteurs s'impose donc, ce que les mots de Sophie Pointurier facilitent, à la manière de ceux de l'interprète. 